\title{
An unusual case of compound naevus of the scalp with hair greying, suggesting melanoma in dermoscopy
}

\author{
Grazyna Kaminska-Winciorek ${ }^{1}$, Iris Zalaudek², Zdzislaw Wozniak³ ${ }^{3}$, Maciej Baglaj ${ }^{4}$
} 1Department of Bone Marrow Transplantation and Onco-Hematology, Maria Sklodowska-Curie National Research Institute of Oncology
(MSCNRIO) Gliwice Branch, Gliwice, Poland
2Department of Dermatology and Venereology, University of Trieste, Ospedale Maggiore, Trieste, Italy
${ }^{3}$ Department of Pathology, Wroclaw Medical University, Wroclaw, Poland
${ }^{4}$ Department of Paediatric Surgery and Urology, Wroclaw Medical University, Wroclaw, Poland

Adv Dermatol Allergol 2020; XXXVII (2): 274-276 DOI: https://doi.org/10.5114/ada.2020.94848

Based on clinical and dermoscopic morphology, scalp naevi can be divided into six main groups, namely common, papillomatous, eclipse, congenital, blue and atypical naevi [1]. For the latter, histopathological diagnosis is mandatory to rule out melanoma.

Herein we present a case of an unusual clinical and dermoscopic presentation of a scalp naevus resulting in skin and hair depigmentation.

An 11-year-old girl was referred to our clinic because of a recent onset of hair greying within a pre-existing congenital scalp naevus located in the occipito-parietal region. Moreover, there was a history of slow enlargement and development of the nodular area within the naevus two years before. Clinically, a flat pigmented lesion with grey hair, measuring $5 \times 2 \mathrm{~cm}$ with three firm white and bluish nodules measuring $5 \mathrm{~mm}$ in diameter was seen (Figure 1 A). Grey hair was growing within the entire lesion (Figure $1 \mathrm{~B}$ ). Dermoscopy revealed a structureless pattern with white and grey colours suggesting melanoma (Figure $1 \mathrm{C}$ ). At the periphery some brown globules (clods) were noted. To rule out melanoma arising in the naevus, a biopsy of the nodular area was performed. Histopathological examination revealed a compound naevus with congenital features.

Because of the unusual presentation, the naevus was excised in two steps and histopathology confirmed the diagnosis of a compound naevus. Histopathology revealed an uneven distribution and heterogeneous cellular density of melanocytes with irregular distribution of melanin (intra- and extracellular distribution). Foci of homogenisation of the connective tissue stroma were visible in the upper dermal layers; there were also areas of fibrosis and scarring of the connective tissue stroma deeper in the dermis (mainly around the hair follicles) (Figure 1 D). Melan A immunohistochemistry showed a normal or increased melanocyte count in the stratum basale of the epidermis, and a significantly decreased number of melanocytes or complete lack of these cells in the hair follicles (Figure $1 \mathrm{E}$ ). This finding may be considered the cause of the discoloured hairs. Melanin granules were detected through Fontana-Masson histochemical staining (Figure $1 \mathrm{~F}$ ).

Changes in the pigmentation of naevi located within the scalp may include more intense coloration (physiologically or in the case of malignant transformation), as well as the loss of pigment as in e.g. Sutton naevus, dying naevus, melanoma and during metastatic melanoma immunotherapy [2, 3]. Classic melanocytic naevi in children manifesting clinically as solid-coloured lesions very rarely show a homogenous pattern (only in 6\%) [4]. Usually, globular (57\%) and complex (reticular-globular) (27\%) dermoscopic patterns dominate; reticular (9\%) and fibrillar (1\%) are rare [4]. Perifollicular hypopigmentation, causing the appearance of irregular borders, is the unifying feature of the majority of children scalp naevi [4].

Furthermore, among scalp naevi in children, stereotypical eclipse naevi are often reported $[4,5]$. In childhood they manifest as flat blotch with central hypopigmentation [1]. During adolescence the central area may become more elevated, and over time the peripheral pigmentation disappears, finally taking the form of a raised to nodular, hypopigmented naevus typical for adults [1]. Moreover, the frequency of eclipse naevi decreases with age from a reported level of $20.5 \%$ [4, 5] in a paediatric study population to $14.5 \%$ (mean age 25 years) in Zalaudek et al. study [1].

Address for correspondence: Grażyna Kamińska-Winciorek MD, PhD, Associate Prof., Department of Bone Marrow Transplantation and Onco-Hematology, Maria Sklodowska-Curie National Research Institute of Oncology (MSCNRIO), Gliwice Branch, 15 Wybrzeże Armii Krajowej St, 44-101 Gliwice, Poland, phone: +48 604070 208, e-mail: dermatolog.pl@gmail.com Received: 15.01.2020, accepted: 25.01.2020. 

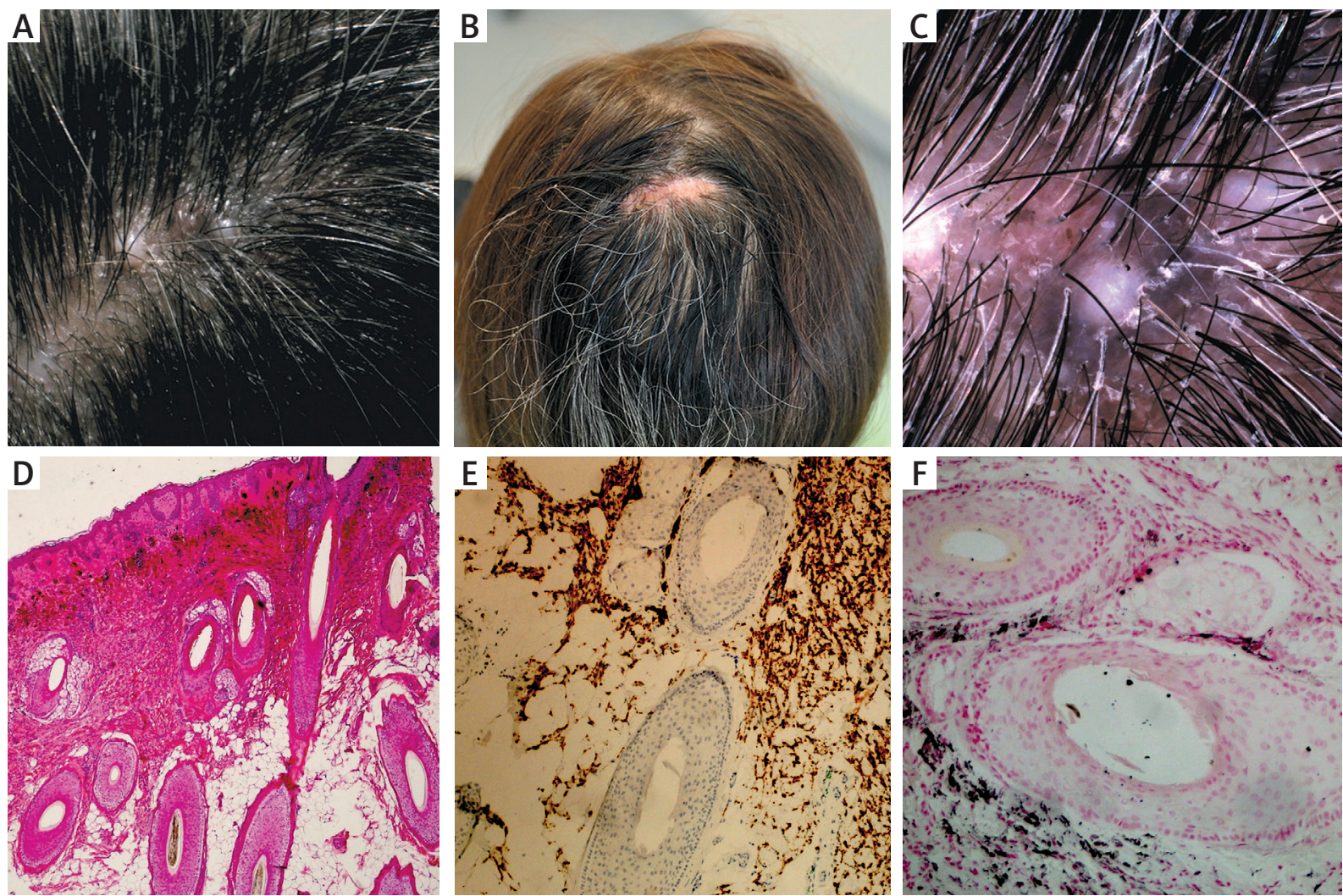

Figure 1. A - Clinical presentation of the compound naevus of the scalp occurring as a flat pigmented lesion with grey hair, measuring $5 \times 2 \mathrm{~cm}$ with three firm, white and bluish nodules measuring $5 \mathrm{~mm}$ in diameter. B - Clinical presentation of the macroscopic picture of the compound naevus of the scalp with the presence of greyish, silver hair growing out of naevus. C - Dermoscopy in polarized light (DermliteCam, 3Gen, San Juan Capistrano, California, USA) reveals a structureless pattern with the white and grey colours. At the periphery some brown globules (clods) are noted. Grey hair is growing within the entire lesion. D - Uneven distribution and heterogeneous cellular density of melanocytes with irregular distribution of melanin (intra- and extracellular distribution); fibrosis and scarring around the hair follicles) (H\&E, 100x magnification). E - Melan A immunohistochemistry: a significantly decreased number of melanocytes or complete lack of these cells in the hair follicles (200x magnification). F - Fontana-Masson histochemical staining: a decreased number or complete lack of melanin granules in the hair follicles (200x magnification)

The occurrence of grey or white colour that indicates regression always requires the exclusion of melanoma, including metastatic melanoma, as well as blue nevi. According to a multicentre study of the International Dermoscopy Society by Stanganelli et al. [5], approximately one-fourth of all scalp naevi (25.7\%) were blue naevi. Dermoscopy indicated the presence of structureless blue pigmentation and additional areas of hypopigmentation [5] (Figures 2 A, B).

Regression and atypical network/pseudonetwork may occur in melanoma in situ and thin scalp melanomas [5]. Thick melanomas present dermoscopically unspecific patterns (Figures 2 C, D), blue white veil, irregular dots and black blotches [5]. Dermoscopic features of scalp melanoma clearly differ from facial melanoma revealing the same pattern as seen in melanoma of the trunk including regression structures (white scar-like areas) [6].

What is more, the differential diagnosis of black- and grey-coloured lesions should include pigmented basal cell carcinoma (BCC) with its dermoscopic clues such as leaflike structures, blue-grey ovoid/globular structures, spoke wheel structures, arborizing vessels, ulceration/multiple erosions. Scalp BCCs have significantly more pigmentation and melanocytic criteria than BCCs located elsewhere [7]. Furthermore, angiosarcoma should be ruled out as its dermoscopic manifestations include multiple, homogenous, structureless, whitish-pink areas with white lines, multiple structureless areas as a combination of red, purple and blue colour with accompanying white "steam-like" areas [8, 9].

Our case suggests that scalp naevi may undergo spontaneous changes even in the absence of malignant transformation. Although we are unable to explain the loss of cells within the hair follicles, it appears to be the cause of sudden hair greying in the presented patient. Inzinger et al. [10] previously described a 91-year-old woman with progressive black repigmentation of otherwise completely white hair at the same site within the melanoma of the scalp. 

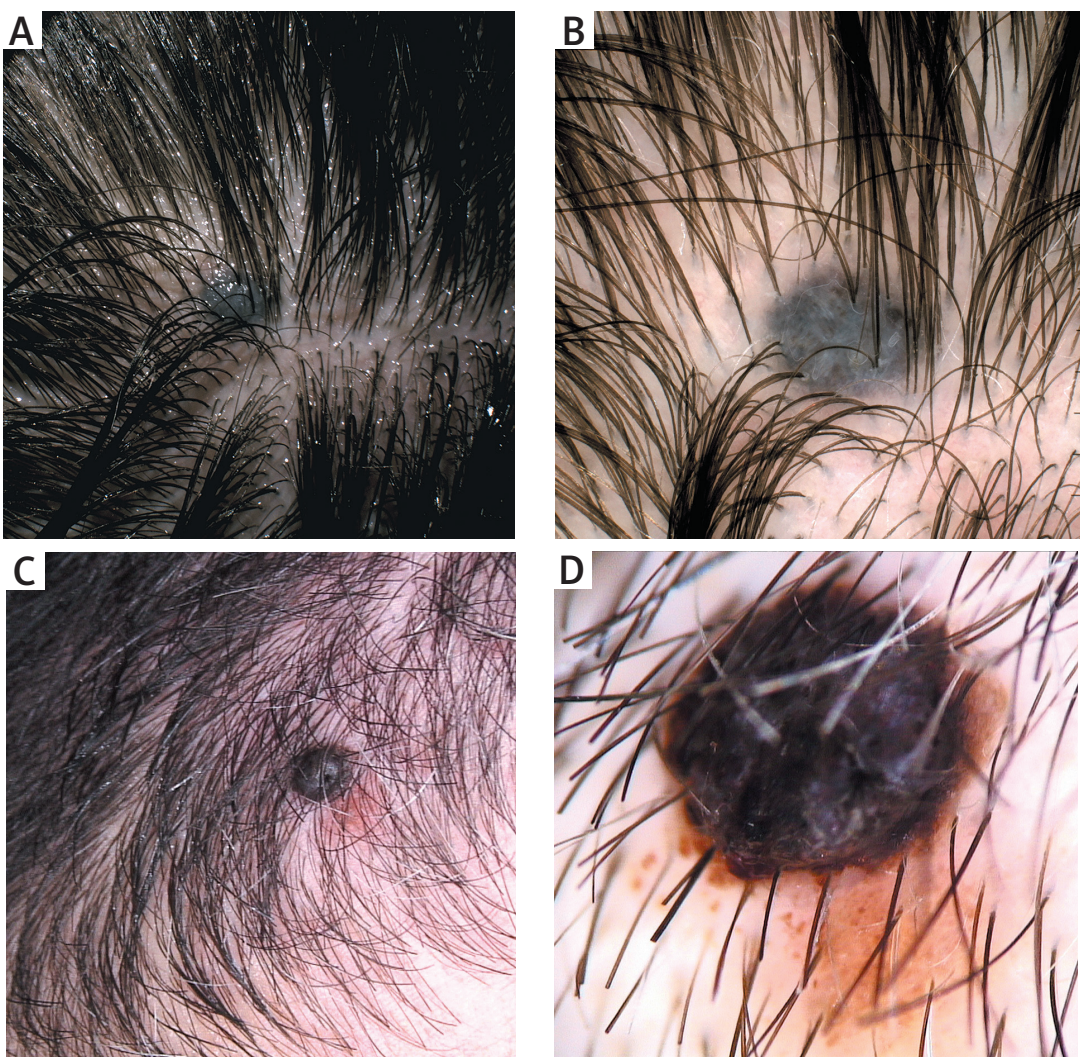

Figure 2. A - Macroscopic picture of a nodular bluish-blackish lesion of $2 \mathrm{~mm}$ in diameter within the occipital region of the scalp in a 40-year-old woman. Because of unknown history the lesion was surgically excised and proved histopathologically as blue naevus. B - Dermoscopic picture of the blue naevus of the scalp in polarized light (DermliteCam, 3Gen, San Juan Capistrano, California, USA) revealed the structureless pattern with superficial whitish crystalline streaks arranged in a non-specific pattern. No change in hair colour is noted. C - Clinical presentation of $5 \mathrm{~mm}$ nodule with rapid growth in a 40-year-old man. After total surgical excision the histopathology proved melanoma, Breslow $0.45 \mathrm{~mm}$ that arose within pre-existing melanocytic naevus. D - Dermoscopic picture of the scalp nodular melanoma in polarized light (DermliteCam, 3Gen, San Juan Capistrano, California, USA) shows a structureless pattern with focal whitish crystalline streaks resembling chrysalids. In the lower part of the lesion, brown clods can be seen, suggesting residual melanocytic naevus. In the central part of the melanoma hair greying is noted

\section{Conflict of interest}

The authors declare no conflict of interest.

\section{References}

1. Zalaudek I, Schmid K, Niederkorn A, et al. Proposal for a clinical-dermoscopic classification of scalp naevi. Br J Dermatol 2014; 170: 1065-72.

2. Zalaudek I, Donati P, Catricalà C, Argenziano G. ["Dying nevus” or regressing melanoma]. Hautarzt 2011; 62: 293-6.

3. Wolner ZJ, Marghoob AA, Pulitzer MP, et al. A case report of disappearing pigmented skin lesions associated with pembrolizumab treatment for metastatic melanoma. $\mathrm{Br}$ J Dermatol 2018; 178: 265-9.

4. Tcheung WJ, Bellet JS, Prose NS, et al. Clinical and dermoscopic features of 88 scalp naevi in 39 children. Br J Dermatol 2011; 165: 137-43.

5. Stanganelli I, Argenziano G, Sera F, et al. Dermoscopy of scalp tumours: a multi-centre study conducted by the international dermoscopy society. J Eur Acad Dermatol Venereol 2012; 26: 953-63.
6. Zalaudek I, Leinweber B, Soyer HP, et al. Dermoscopic features of melanoma on the scalp. J Am Acad Dermatol 2004; 51 (2 Suppl): S88-90.

7. Suppa M, Micantonio T, Di Stefani A, et al. Dermoscopic variability of basal cell carcinoma according to clinical type and anatomic location. J Eur Acad Dermatol Venereol 2015; 29: 1732-41.

8. Zalaudek I, Gomez-Moyano E, Landi C, et al. Clinical, dermoscopic and histopathological features of spontaneous scalp or face and radiotherapy-induced angiosarcoma. Australas J Dermatol 2013; 54: 201-7.

9. Oiso N, Matsuda H, Kawada A. Various colour gradations as a dermatoscopic feature of cutaneous angiosarcoma of the scalp. Australas J Dermatol 2013; 54: 36-8.

10. Inzinger M, Massone C, Arzberger E, Hofmann-Wellenhof R. Hair repigmentation in melanoma. Lancet 2013; 382: 1224. 